\title{
Resolução de Problemas de Escoamento em Meios Porosos Heterogêneos em Geometrias Complexas com o Método de Galerkin Descontínuo
}

\author{
Igor Mozolevski, Luciane I. A. Schuh \\ UFSC - Departamento de Matemática, \\ Campus Trindade, 88040-900, Florianópolis, SC \\ E-mail: igor.e.mozolevski@gmail.com, luciane.schuh@ufsc.br.
}

\begin{abstract}
Resumo: Empregamos o método de Galerkin descontínuo ( $D G$ ) para resolução numérica de problemas de escoamento em meios porosos heterogêneos e geometrias complexas com a presença de forças capilares descontínuas. As heterogeneidades nas propriedades físicas do meio poroso, como permeabilidade e porosidade, podem provocar o surgimento de forças capilares descontínuas na interface formada entre dois tipos de sedimentos, o que torna necessária a imposição de condições de interface não-lineares no método empregado para simulação numérica de tais problemas. Resolvemos o sistema pressão-saturação com o método de Galerkin descontínuo em que usamos o método de Euler implícito para discretização temporal, fluxo de Godunov para o termo advectivo e as técnicas de média ponderada e média harmônica para lidar adequadamente com os termos degenerados e com as descontinuidades nos coeficientes e na solução. Efetuamos a reconstrução de fluxos no espaço de Raviart-Thomas-Nédélec e impomos fracamente as condições de interface nãolineares. Apresentamos resultados numéricos que ilustram a eficiência e o potencial do método para modelar problemas de escoamento em meios porosos heterogêneos com geometrias complexas.
\end{abstract}

Palavras-chave: Galerkin Descontínuo, Meios Heterogêneos, Pressão Capilar Descontínua, Condições de Interface.

\section{Introdução}

Nos últimos anos ocorreu um significativo aumento na modelagem numérica de problemas de escoamento multifásico com aplicações em diferentes áreas como engenharia de reservatório de petróleo, engenharia química, engenharia ambiental, hidrologia, entre outras. Problemas de escoamento que vem recebendo atenção especial estão relacionados a eliminação de resíduos radioativos e ao sequestro geológico de carbono. As heterogeneidades do meio poroso tem forte impacto sobre o processo de escoamento de fluídos, pois podem provocar o surgimento de forças capilares descontínuas na interface formada entre dois tipos de sedimentos $[1,8,6]$. Neste trabalho empregamos o método de Galerkin descontínuo (DG) para estudar os efeitos das heterogeneidades e forças capilares descontínuas em problemas de escoamento bifásico em meios porosos com geometrias complexas. A importância de simulações numéricas em problemas de escoamento com a presença de forças capilares descontinuas também foi evidenciada no congresso SIAM - Conference on Mathematical and Computational Issues in the Geosciences, realizado em junho de 2013, onde este assunto foi tema de um mini-simposio, em que foram apresentados resultados numericos obtidos com o método aqui empregado.

Consideramos $\Omega$ um domínio em $\mathbb{R}^{d}, d \geq 1$, limitado e aberto, com fronteira $\partial \Omega$ e normal exterior $\eta$. Dividimos o domínio $\Omega$ em subdomínios $\Omega^{(\beta)}$ tal que $\bar{\Omega}=\bigcup_{i=1}^{N_{l}} \overline{\Omega^{(i)}}, N_{l} \geq 2, \Omega^{(i)} \cap \Omega^{(j)}=\emptyset$, $i \neq j$ e $\Gamma^{(i j)}$ a interface formada entre dois subdomínios vizinhos $\Omega^{(i)}$ e $\Omega^{(j)}$. A Cada subdomínio $\Omega^{(\beta)}, \beta \in\left\{1,2, \ldots, N_{l}\right\}$, associamos as constantes de porosidade $\Phi^{(\beta)}$ e permeabilidade $\mathrm{K}^{(\beta)}$. De forma geral, para qualquer função real $u$ definida em $\Omega$, denotamos sua restrição à $\Omega^{(\beta)}$ por $u^{(\beta)}$. Desta forma o processo de escoamento bifásico, incompressível em meios porosos heterogêneos em cada subdomínio $\Omega^{(\beta)}, \beta \in\left\{1,2, \ldots, N_{l}\right\}$, pode ser descrito pelo sistema de equações diferenciais parciais: 


$$
\begin{aligned}
-\nabla \cdot\left(k^{(\beta)}(s) \nabla p\right) & =F_{\omega}^{(\beta)}+F_{n}^{(\beta)}, \\
\mathbf{q}^{(\beta)} & =-k^{(\beta)}(s) \nabla p, \\
\left.\Phi^{(\beta)} \partial_{t} s+\nabla \cdot\left(\mathbf{q}^{(\beta)} f_{n}^{(\beta)}(s)-\epsilon^{(\beta)}(s) \nabla s\right)\right) & =F_{n}^{(\beta)},
\end{aligned}
$$

onde $p$, q e $s$ são pressão global, velocidade total e saturação da fase não-molhante, respectivamente, sendo que omitimos o índice $\beta$ em $s$ e $p$. Denotamos a mobilidade da fase molhante $(\alpha=\omega)$ e da fase não-molhante $(\alpha=n)$ por $\lambda_{\alpha}^{(\beta)}=k r_{\alpha}^{(\beta)} / \mu_{\alpha}$, sendo $k r_{\alpha}^{\beta}$ a permeabilidade relativa e $\mu_{\alpha}$ é a viscosidade do fluido, a soma $\lambda^{(\beta)}:=\lambda_{\omega}^{(\beta)}+\lambda_{n}^{(\beta)}$ como a mobilidade total, $f_{\alpha}^{(\beta)}:=\frac{\lambda_{\alpha}^{(\beta)}}{\lambda^{(\beta)}}$ o fluxo fracionário, $k^{(\beta)}=\lambda^{(\beta)} \mathrm{K}^{(\beta)}, \epsilon^{(\beta)}=\lambda_{\omega}^{(\beta)} f_{n}^{(\beta)} K^{(\beta)}\left(\pi^{(\beta)}\right)^{\prime}$, onde $\pi^{(\beta)}$ é a pressão capilar e $F_{\alpha}^{(\beta)}$ os termos de fonte correspondentes a cada fase. Assumimos que $s \in\left[S_{n r}^{(\beta)}, 1-S_{w r}^{(\beta)}\right]$, sendo $S_{w r}$ e $S_{n r}$ as saturações residuaias da fase molhante e não-molhante, respectivamente. Denotamos a saturação efetiva da fase não-molhante por $s_{n e}^{(\beta)}(s)=\left(s^{(\beta)}-s_{n r}^{(\beta)}\right) /\left(1-s_{n r}^{(\beta)}-s_{\omega r}^{(\beta)}\right)$.

Para completar o sistema consideramos condições de fronteira e iniciais e condições de interface conforme descrevemos a seguir. Sumpomos que $\partial \Omega=\partial \Omega_{D} \cup \partial \Omega_{N}$. As condições de fronteira para pressão e saturação podem ser de Dirichlet $p=p_{D}, \quad s=s_{D}$ em $\partial \Omega_{D}$ ou Neumann $\mathbf{q}(p, s) \cdot \eta=0, \quad \mathbf{r}(p, s ; s) \cdot \eta=0 \quad$ em $\partial \Omega_{N}$, onde $\mathbf{r}^{(\beta)}(p, \xi ; s)=-\epsilon^{(\beta)}(s) \nabla s+\mathbf{q}^{(\beta)}(p, \xi) f_{n}^{(\beta)}(s)$ é o fluxo volumétrico da saturação. Como condição inicial consideramos $s^{(\beta)}=s_{0}^{(\beta)}$. As Eqs. (1)-(3) são válidas somente em $\Omega^{(\beta)}, \beta \in\left\{1,2, \ldots, N_{l}\right\}$ e na interface $\Gamma^{(i j)}$, em que porosidade, permeabilidade e forças capilares são descontínuas, estas equações deixam de ser válidas. Logo precisamos de duas condições para serem impostas em $\Gamma^{(i j)}$, que são as condições de interface. A imposição das condições de interface torna-se indispensável na modelagem de problemas de escomento em meios porosos com diferentes pressões de entrada $P_{e}^{(\beta)}$, pressão necessária para um fluido não-molhante escoar através de um meio inicialmente saturado por um fluido molhante. Para mais detalhes ver $[8,3]$. Descrevemos as condições de interface para $\Gamma=\partial \Omega^{(1)} \cap \partial \Omega^{(2)}$, com $P_{e}^{(1)}<P_{e}^{(2)}$ e $\pi^{(1)}(s)<\pi^{(2)}(s), \forall s \in \cap_{\beta=1,2}\left[S_{n r}^{(\beta)}, 1-S_{\omega r}^{(\beta)}\right)$. Para definir as condições de interface introduzimos as funções salto para saturação e pressão global:

$$
\begin{aligned}
& j_{s}(\xi)=\xi-\bar{\pi}^{(2)}\left(\pi^{(1)}(\xi)\right), \quad \text { se } \xi \in\left[s_{n r}^{(1)}, 1-s_{w r}^{(1)}\right) \\
& j_{p}(\xi)= \begin{cases}\int_{s_{n}^{(1)}\left(f_{n}\right)}^{\xi}(1)\left(\pi^{(1)}\right)^{\prime}+P_{e}^{(1)}-P_{e}^{(2)}, & \text { se } \xi \in\left[s_{n r}^{(1)}, s^{*}\right), \\
\left.\int_{s_{n r}^{(2)}}^{(2)}(\xi)\right) f_{\omega}^{(2)}\left(\pi^{(2)}\right)^{\prime}-\int_{s_{n r}^{(1)}}^{\xi} f_{w}^{(1)}\left(\pi^{(1)}\right)^{\prime}, & \text { se } \xi \in\left[s^{*}, 1-s_{\omega r}^{(1)}\right),\end{cases}
\end{aligned}
$$

em que $s^{*} \in\left(s_{n r}^{(1)}, 1-s_{\omega r}^{(1)}\right)$ representa a saturação de entrada definida tal que $\pi^{(1)}\left(s^{*}\right)=\pi^{(2)}\left(s_{n r}^{(2)}\right)$ e $\bar{\pi}^{(\beta)}$ a extensão da inversa de $\pi^{(\beta)}$ :

$$
\bar{\pi}^{\beta}(p)=\left\{\begin{array}{cl}
s_{n r}^{(\beta)}, & \text { se } p<P_{e}^{(\beta)} \\
\left(\pi^{(\beta)}\right)^{-1}(p), & \text { se } p \geq P_{e}^{(\beta)}
\end{array}\right.
$$

Desta forma as condições de interface que devem ser impostas sobre $\Gamma=\partial \Omega^{(1)} \cap \partial \Omega^{(2)}$ para saturação e pressão são, respectivamente:

$$
\left\{\begin{array} { l } 
{ \mathbf { r } ^ { ( 1 ) } ( p ^ { ( 1 ) } , s ^ { ( 1 ) } ; s ^ { ( 1 ) } ) \cdot \eta _ { \Gamma } = \mathbf { r } ^ { ( 2 ) } ( p ^ { ( 2 ) } , s ^ { ( 2 ) } ; s ^ { ( 2 ) } ) \cdot \eta _ { \Gamma } } \\
{ s ^ { ( 1 ) } - s ^ { ( 2 ) } = j _ { s } ( s ^ { ( 1 ) } ) }
\end{array} \quad \left\{\begin{array}{l}
\mathbf{q}^{(1)}\left(p^{(1)}, s^{(1)}\right) \cdot \eta_{\Gamma}=\mathbf{q}^{(2)}\left(p^{(2)}, s^{(2)}\right) \cdot \eta_{\Gamma} \\
p^{(1)}-p^{(2)}=j_{p}\left(s^{(1)}\right) .
\end{array}\right.\right.
$$

Para mais detalhes relacionados à formulação do problema e às condições de interface ver $[4,7]$.

\section{O Método de Galerkin Descontínuo}

Para discretização espacial particionamos o domínio $\Omega$ em elementos triangulares $\{\mathcal{T}\}_{h>0}$. Seja $h_{T}$ o diâmetro do elemento $T$ e $h=\max _{T \in \mathcal{T}} h_{T}$ o diâmetro da malha. Consideramos $\mathcal{E}_{h}$ o conjunto formado por todas as faces, sendo $h_{E}$ o diâmetro da face $E$ e $\mathcal{E}_{h}=\mathcal{E}_{h}^{\partial} \cup \mathcal{E}_{h}^{\mathrm{i}}$, onde $\mathcal{E}_{h}^{\partial}=\mathcal{E}_{h}^{\partial \mathrm{D}} \cup \mathcal{E}_{h}^{\partial \mathrm{N}}$, sendo $\mathcal{E}_{h}^{\partial \mathrm{D}}, \mathcal{E}_{h}^{\partial \mathrm{N}}$ as faces que cobrem $\partial \Omega_{D} e \partial \Omega_{N}$ e $\mathcal{E}_{h}^{\mathrm{i}}$ as faces interiores. Assumimos também que as interfaces $\Gamma$ são exatamente cobertas por um conjunto de faces armazenadas em $\mathcal{E}_{h}^{\Gamma}$. Para a discretização temporal consideramos a sequencia de tempos $\left\{t^{m}\right\}_{0 \leq m \leq M}$ tal que $t^{0}=0, t^{M}=T$ e passo no tempo $\tau_{m}=t^{m}-t^{m-1}, m=1, \ldots, M$,. 
Para uma função $v$, definimos o salto e a média em $E \in \mathcal{E}_{h}^{\text {i }}$ em que $E=\partial T^{-} \cap \partial T^{+}$: $\llbracket v \rrbracket:=\left.v^{-}\right|_{E}-\left.v^{+}\right|_{E}, \quad\{\{v\}\}:=\frac{\left.v^{-}\right|_{E}+\left.v^{+}\right|_{E}}{2}, \quad$ onde $v^{ \pm}:=\left.v\right|_{T^{ \pm}}$, extendemos essas definições para as faces da fronteira $E \in \mathcal{E}_{h}^{\partial}$ como $\llbracket v \rrbracket=\{\{v\}\}=\left.v\right|_{E}$. Para a face $E$ também definimos a normal $n_{E}$ como sendo exterior ao elemento $T^{-}$e para uma face de fronteira $E \in \mathcal{E}_{h}^{\partial}$, a normal $n_{E}=\eta$. Para lidar com as descontinuidades e coeficientes degenerados do termo difusivo no sistema de equações (1)-(3), introduzimos a média ponderada e a média harmônica de acordo com $[2,5]$. Considere uma função escalar $a$, representando a difusividade definida em uma face $E \in \mathcal{E}_{h}^{\mathrm{i}}$, com valores $a_{T^{-}, E}$ e $a_{T^{+}, E}$ associados à $T^{-}$e $T^{+}$, respectivamente. Então na face $E \in \mathcal{E}_{h}^{\mathrm{i}}$ definimos, respectivamente, a média ponderada para uma função $v$ e a média harmônica de $a$ por,

$$
\begin{aligned}
\{\{v\}\}_{a} & :=\omega_{T^{-}, E}(a) v^{-}+\omega_{T^{+}, E}(a) v^{+}, \text {onde } \\
\omega_{T^{-}, E}(a) & :=\frac{\left\|a_{T^{+}, E}\right\|_{L^{\infty}(E)}}{\left\|a_{T^{-}, E}\right\|_{L^{\infty}(E)}+\left\|a_{T^{+}, E}\right\|_{L^{\infty}(E)}}, \omega_{T^{+}, E}(a):=\frac{\left\|a_{T^{-}, E}\right\|_{L^{\infty}(E)}}{\left\|a_{T^{-}, E}\right\|_{L^{\infty}(E)}+\left\|a_{T^{+}, E}\right\|_{L^{\infty}(E)}} \\
\langle a\rangle_{E} & :=\frac{2\left\|a_{T^{-}, E}\right\|_{L^{\infty}(E)}\left\|a_{T^{+}, E}\right\|_{L^{\infty}(E)}}{\left\|a_{T^{-}, E}\right\|_{L^{\infty}(E)}+\left\|a_{T^{+}, E}\right\|_{L^{\infty}(E)}} \quad\langle a\rangle_{\left.E\right|_{\mathcal{E}_{h}^{\Gamma}}}=\frac{2\|a\|_{L^{\infty}\left(\Omega^{\left(\beta_{1}\right)}\right)}\|a\|_{L^{\infty}\left(\Omega^{\left(\beta_{2}\right)}\right)}}{\|a\|_{L^{\infty}\left(\Omega^{\left(\beta_{1}\right)}\right)}+\|a\|_{L^{\infty}\left(\Omega^{\left(\beta_{2}\right)}\right)}}
\end{aligned}
$$

onde os pesos $\omega_{T^{-}, E}$ e $\omega_{T^{+}, E}$, são definidos tal que $\omega_{T^{-, E}}(a)+\omega_{T^{+}, E}(a)=1$. Para faces na fronteira extendemos as definições acima por $\{\{v\}\}_{a}:=v$ e $\langle a\rangle_{E}:=\|a\|_{L^{\infty}(E)}$. Se $\left\|a_{T^{-}, E}\right\|_{L^{\infty}(E)}$. $\left\|a_{T^{+}, E}\right\|_{L^{\infty}(E)}=0$ em $E$, tomamos $\{\{v\}\}_{a}=\{\{v\}\}$ e $\langle a\rangle_{E}=\|a\|_{L^{\infty}\left(\Omega^{\beta}\right)}$ para $E \in \Omega^{\beta}$.

Seja $k \geq 1$ um inteiro. Procuramos aproximações no instante de tempo $t^{m}$ para a pressão $p_{h}^{m}$ e para a saturação $s_{h}^{m}$ no espaço de elementos finitos descontínuo: $V_{h}^{k}:=\left\{v_{h} \in L^{2}(\Omega) ; \forall T \in\right.$ $\left.\mathcal{T},\left.v_{h}\right|_{T} \in \mathbb{P}_{k}(T)\right\}$, onde $\mathbb{P}_{k}(T)$ é o espaço de aproximação polinomial de grau $\leq k$ sobre $T$. Passamos agora a descrever o método de Galerkin descontínuo sequencial usado para resolver o sistema acoplado pressão-saturação (1)-(3).

Dada $s_{h}^{m-1} \in V_{h}^{k}, 1 \leq m \leq M$ do passo no tempo anterior $(m \geq 1)$, resolvemos a equação para a pressão (1) usando o método DG com penalização interior, ou seja, procuramos $p_{h}^{m} \in V_{h}^{k}$ tal que $\forall z \in V_{h}^{k}$,

$$
\begin{aligned}
& \sum_{T \in \mathcal{T}} \int_{T} \kappa\left(s_{h}^{m-1}\right) \nabla p_{h}^{m} \cdot \nabla v_{h}+\sum_{E \in \mathcal{E}_{h}^{\mathrm{i}} \cup \mathcal{E}_{h}^{\partial \mathrm{D}}}\left\langle\kappa\left(s_{h}^{m-1}\right)\right\rangle_{E} \frac{\sigma_{E} k^{2}}{h_{E}} \int_{E} \llbracket p_{h}^{m} \rrbracket \llbracket v_{h} \rrbracket \\
& -\sum_{E \in \mathcal{E}_{h}^{\mathrm{i} \cup \mathcal{E}_{h}^{\partial \mathrm{D}}}} \int_{E}\left(\left\{\left\{n_{E} \cdot \kappa\left(s_{h}^{m-1}\right) \nabla p_{h}^{m}\right\}\right\}_{\kappa\left(s_{h}^{m-1}\right)} \llbracket v_{h} \rrbracket+\vartheta\left\{\left\{n_{E} \cdot \kappa\left(s_{h}^{m-1}\right) \nabla v_{h}\right\}\right\}_{\kappa\left(s_{h}^{m-1}\right)} \llbracket p_{h}^{m} \rrbracket\right) \\
& =\sum_{T \in \mathcal{T}} \int_{T}\left(F_{w}^{m}+F_{n}^{m}\right) v_{h}+\sum_{E \in \mathcal{E}_{h}^{\partial \mathrm{D}}} \int_{E}\left(-\vartheta n_{E} \cdot \kappa\left(s_{h}^{m-1}\right) \nabla v_{h}+\left\langle\kappa\left(s_{h}^{m-1}\right)\right\rangle_{E} \frac{\sigma_{E} k^{2}}{h_{E}} v_{h}\right) p_{D}^{m} \\
& +\sum_{E \in \mathcal{E}_{h}^{\Gamma}} \int_{E}\left(-\vartheta\left\{\left\{n_{E} \cdot \kappa\left(s_{h}^{m-1}\right) \nabla v_{h}\right\}\right\}_{\kappa\left(s_{h}^{m-1}\right)}+\left\langle\kappa\left(s_{h}^{m-1}\right)\right\rangle_{E} \frac{\sigma_{E} k^{2}}{h_{E}} \llbracket v_{h} \rrbracket\right) j_{p}\left(\chi_{E}^{1}\left(s_{h}^{m}\right)\right) .
\end{aligned}
$$

onde $\vartheta$ permite migrar entre as versões simétrica $(=1)$ e não simétrica $(=-1), 0<\sigma_{E}<10$, e a função $\chi_{E}^{1}$ em $V_{h}^{k}$ é definida por $\chi_{E}^{1}\left(v_{h}\right)= \begin{cases}\left.v_{h}^{+}\right|_{E}, & \text { if } T^{+} \subset \Omega^{1}, \\ \left.v_{h}^{-}\right|_{E}, & \text { if } T^{-} \subset \Omega^{1} .\end{cases}$

Calculamos a aproximação $p_{h}^{m}$ para pressão em $V_{h}^{k}$, e assim a componente normal de $\mathbf{q}_{h}^{m}=$ $\kappa^{\beta}\left(s^{m-1}\right) \nabla p^{m}$, definida localmente em cada elemento da malha $T$, não será contínua nas faces $E \in \mathcal{E}_{h}^{\text {i }}$. Para contornar esta dificuldade, reconstruimos esse fluxo no espaço de elementos finitos de Raviart-Thomas-Nédélec tal que o fluxo reconstruído $\mathbf{u}_{h}^{m}$ satisfaça $\nabla \cdot \mathbf{u}_{h}^{m}=F_{w}^{m}+F_{n}^{m}$. Para mais detalhes ver $[4,7]$.

Em seguida, para calcular $s_{h}^{m}$, usamos o método de Euler implícito no tempo, juntamente com uma discretização espacial do termo difusivo pelo método DG com penalização interior e fluxo de Godunov para o termo advectivo não-linear. Consequentemente, dados $p_{h}^{m} \in V_{h}^{k}$ e $\mathbf{u}_{h}^{m} \in \mathbf{R T}_{0}(\mathcal{T})$ 
procuramos $s_{h}^{m} \in V_{h}^{k}$ tal que para todo $z_{h} \in V_{h}^{k}$,

$$
\begin{aligned}
\sum_{T \in \mathcal{T}} \int_{T} \epsilon\left(s_{h}^{m}\right) \nabla s_{h}^{m} \cdot \nabla z_{h}+\sum_{E \in \mathcal{E}_{h}^{\mathrm{i} \cup \mathcal{E}}}\left\langle\epsilon\left(s_{h}^{m-1}\right)\right\rangle_{E} \frac{\sigma_{E} k^{2}}{h_{E}} \int_{E} \llbracket s_{h}^{m} \rrbracket \llbracket z_{h} \rrbracket+\sum_{T \in \mathcal{T}} \int_{T} \Phi \frac{1}{\tau^{m}} s_{h}^{m} z_{h} \\
-\sum_{E \in \mathcal{E}_{h}^{\mathrm{i} \cup \mathcal{E}_{h}^{\partial \mathrm{D}}}} \int_{E}\left(\left\{\left\{n_{E} \cdot \epsilon\left(s_{h}^{m}\right) \nabla s_{h}^{m}\right\}\right\}_{\epsilon\left(s_{h}^{m-1}\right)} \llbracket z_{h} \rrbracket+\vartheta\left\{\left\{n_{E} \cdot \epsilon\left(s_{h}^{m}\right) \nabla z_{h}\right\}\right\}_{\left.\epsilon\left(s_{h}^{m-1}\right) \llbracket s_{h}^{m} \rrbracket\right)}\right. \\
-\sum_{T \in \mathcal{T}} \int_{T} \mathbf{u}_{h}^{m} f_{n}\left(s_{h}^{m}\right) \cdot \nabla z_{h}+\sum_{E \in \mathcal{E}_{h}} \int_{E}\left(\mathbf{u}_{h}^{m} \cdot n_{E}\right) \Psi_{h E}^{m} \llbracket z_{h} \rrbracket+\sum_{E \in \mathcal{E}_{h}^{\partial \mathrm{D}}} \int_{E} \vartheta n_{E} \cdot \epsilon\left(s_{h}^{m}\right) \nabla z_{h} s_{D}^{m} \\
+\sum_{E \in \mathcal{E}_{h}^{\Gamma}} \int_{E}\left(\vartheta\left\{\left\{n_{E} \cdot \epsilon\left(s_{h}^{m}\right) \nabla z_{h}\right\}\right\}_{\epsilon\left(s_{h}^{m-1}\right)}-\left\langle\epsilon\left(s_{h}^{m-1}\right)\right\rangle_{E} \frac{\sigma_{E} k^{2}}{h_{E}} \llbracket z_{h} \rrbracket\right) j_{s}\left(\chi_{E}^{1}\left(s_{h}^{m}\right)\right) \\
=\sum_{T \in \mathcal{T}} \int_{T} \Phi \frac{1}{\tau^{m}} s_{h}^{m-1} z_{h}+\sum_{T \in \mathcal{T}} \int_{T} F_{n}^{m} z_{h}+\sum_{E \in \mathcal{E}_{h}^{\partial \mathrm{D}}} \int_{E}\left\langle\epsilon\left(s_{h}^{m-1}\right)\right\rangle_{E} \frac{\sigma_{E} k^{2}}{h_{E}} z_{h} s_{D}^{m}
\end{aligned}
$$

Onde $\Psi_{h E}^{m}$ é fluxo numérico tipo Godunov. Para resolver o sistema não-linear na equação para a saturação empregamos o método de Newton para o termo advectivo e a linearização de Picard para o termo difusivo. Para mais detalhes consultar [7].

\section{Simulação Numérica}

Empregamos o método de Galerkin descontínuo (10)-(11) na simulação numérica de dois problemas de escoamento em meios porosos heterogêneos com pressão capilar descontínua e geometria complexa aplicados a problemas na engenharia de petróleo.

Exemplo 1 Consideramos o modelo de Brooks-Corey para mobilidades e pressão capilar: $\lambda_{n}^{(\beta)}(s)=\frac{1}{\mu_{n}} s_{n e}^{(\beta)^{2}}\left(1-\left(1-s_{n e}^{(\beta)}\right)^{\frac{2+\theta^{(\beta)}}{\theta^{(\beta)}}}\right), \lambda_{w}^{(\beta)}(s)=\frac{1}{\mu_{w}}\left(1-s_{n e}^{(\beta)}\right)^{\frac{2+3 \theta^{(\beta)}}{\theta^{(\beta)}}}, \pi^{(\beta)}(s)=P_{e}^{(\beta)}\left(1-s_{n e}^{(\beta)}\right)^{\frac{-1}{\theta^{(\beta)}}}$,

com dados descritos na Tab. 1 para os Testes 1 a e 1 b e dominio $\Omega=(0,300)^{2}$, (Fig. 1a) dividido em subdomínios: $\Omega^{(2)}=(93.75,206.25) \times(93.75,206.25), \Omega^{(3)}=(0,150) \times(230,300), \Omega^{(1)}=$ $\Omega \backslash\left(\bar{\Omega}^{(2)} \cup \bar{\Omega}^{(3)}\right)$, viscosidade $\mu_{w}=1 e-3 \mathrm{Kg} / \mathrm{ms}, \quad \mu_{n}=1 e-2 \mathrm{Kg} / \mathrm{ms}$ e $\theta=(2,2,2)$, $s_{n r}=(0,0.25,0), s_{w r}=(0,0,0)$. Condição inicial e condições de fronteira:

$$
\begin{aligned}
& S_{0}(x, y)=\left\{\begin{array}{cl}
0.8, & \text { para }(x, y) \in \Omega_{\text {oil }}(\text { verFig. } 1 b), \\
0.25, & \text { para }(x, y) \in \Omega^{(2)}(\text { verFig. } 1 b), \\
0, & \text { Caso contrário. }
\end{array}\right. \\
& -\left.\eta \cdot \mathbf{q}_{n}^{(1)}\right|_{\partial \Omega^{-}}=0, \quad-\left.\eta \cdot \mathbf{q}_{w}^{1}\right|_{\partial \Omega^{-}}=710^{-6} \mathrm{~m} /\left.\mathrm{s} \quad p\right|_{\partial \Omega^{+}}=1.1610^{5} \mathrm{~Pa},\left.\quad s\right|_{\partial \Omega^{+}}=0 ; \\
& -\left.\eta \cdot \mathbf{q}^{1}\right|_{\partial \Omega_{N}}=0, \quad-\left.\eta \cdot \mathbf{q}_{w}^{1}\right|_{\partial \Omega_{N}}=0 ;
\end{aligned}
$$

onde s representa o óleo a água é a fase não-molhante, $\partial \Omega_{s}^{-}$é a região em que a água é injetada e $\partial \Omega_{s}^{+}$região pela qual temos saída de fluxo do reservatório. Note que associamos permeabilidade $K$, pressão de entrada $P_{e}$ e saturações residuais $S_{n r}, S_{w r}$ diferentes para $\Omega^{(1)}, \Omega^{(2)}$ e $\Omega^{(3)}$.

\begin{tabular}{|c|c||c|c|}
\hline \multicolumn{2}{|c||}{ Teste 1a } & \multicolumn{2}{c|}{ Teste 1b } \\
\hline Par. & Valor & Par. & Valor \\
\hline$\Phi$ & $(0.2,0.2,0.2)(-)$ & $\Phi$ & $(0.2,0.2,0.2)(-)$ \\
$K$ & $\left(10^{-10}, 10^{-11}, 10^{-12}\right) \mathrm{m}^{2}$ & $K$ & $\left(10^{-10}, 10^{-11}, 10^{-9}\right) \mathrm{m}^{2}$ \\
$P_{e}$ & $\left(110^{4}, 1.5810^{4}, 1.8310^{4}\right) \mathrm{Pa}$ & $P_{e}$ & $\left(110^{4}, 1.5810^{4}, 7.0710^{3}\right) \mathrm{Pa}$ \\
\hline
\end{tabular}

Tabela 1: Parametros do meio poroso usados no Exemplo 1.

Nas Figuras 2 e 3 apresentamos aproximações para os dados do Teste 1a e Teste 1b, respectivamente. Na Figura 2 podemos notar que em $t=50$ dias o óleo já alcançou a interface $\Gamma^{(12)}$ e atingiu a saturação de entrada, assim permitindo que o óleo cruze a interface e escoe através de $\Omega^{(2)}$ o que pode ser observado na figuras seguintes $t=165,420,1685$ dias, porém percebemos que apenas uma pequena quantidade de óleo consegue cruzar a interface. Em $t=165$ dias podemos perceber que a frente do fluído alcançou a interface $\Gamma^{(13)}$, porém como a saturação do óleo era 


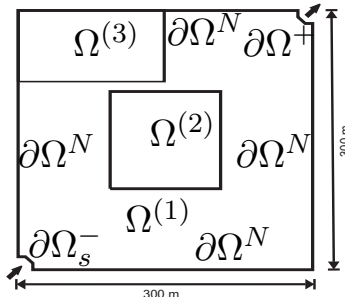

(a)

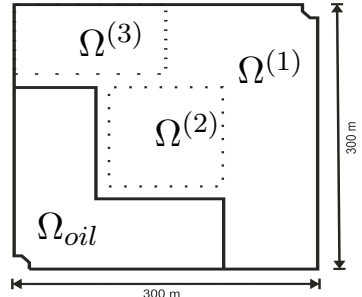

(b)

Figura 1: (a) Domínio $\Omega$ dividido nos subdomínios $\Omega^{(1)}, \Omega^{(2)}$ e $\Omega^{(3)}$ para o Exemplo 1. (b) Domínio $\Omega$ com identificação do subdomínio $\Omega_{o i l}$.

inferior a saturação de entrada o mesmo é conduzido a passar pelo estreito caminho entre $\Omega^{(2)}$ e $\Omega^{(3)}$. Em $t=420$ e $t=1685$ podemos perceber que a maior parte do óleo contorna $\Omega^{(2)}$ e $\Omega^{(3)}$, regiões de baixa permeabilidade. Notamos também que o escoamento sempre ocorre das regiões de maior pressão para regiões de baixa pressão, o que também pode ser observado pelo campo de velocidades.

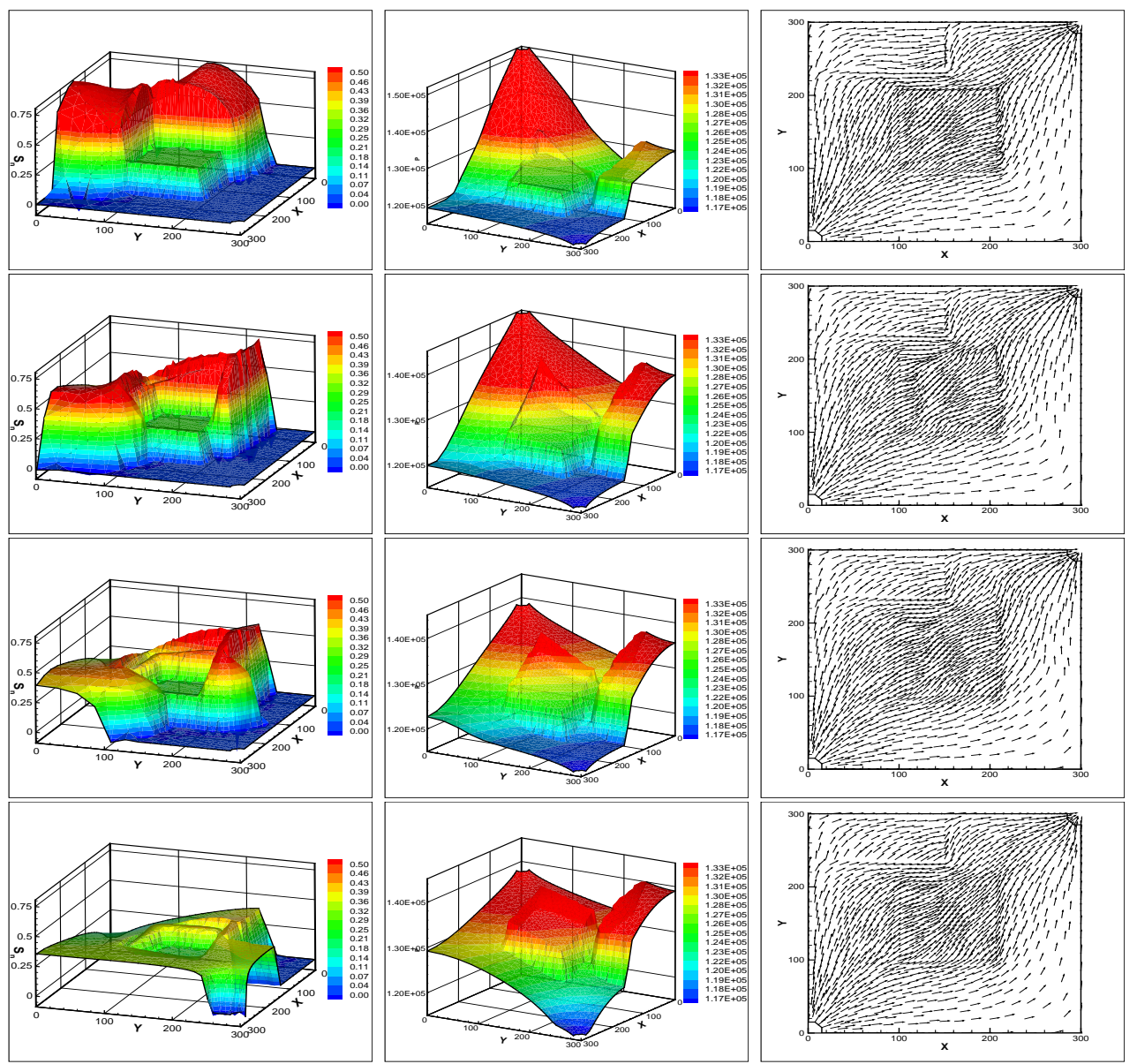

Figura 2: Saturação, pressão global e velocidade total para o Teste 1a. Primeira lina : $t=50$ dias; segunda linha: $t=165$ dias; terceira linha: $t=420$ dias e quarta linha: $t=1685$ dias.

No Teste $1 \mathrm{~b}$, diferentemente do Teste $1 \mathrm{a}$, o subdomínio $\Omega^{(3)}$ possui permeabilidade mais alta e saturação de entrada inferior ao subdomínio $\Omega^{(1)}$, o que está refletido na Figura 3 . Em $t=120$ dias podemos notar que o óleo não teve dificuldade de cruzar a interface $\Gamma^{(13)}$, e devido a maior facilidade de escoamento que o fluido encontra nessa região podemos notar o efeito de sucção nesta interface. Porém em $t=600,4150$ dias podemos perceber que o óleo que cruzou a interface $\Gamma^{(13)}$ ficou acumulado em $\Omega^{(3)}$, pois o mesmo não alcançou a pressão necessária para cruzar novamente a interface $\Gamma^{(13)}$, mas agora pela outra lateral, e escoar através de $\Omega^{(1)}$. Em $t=4150$ dias notamos que parte do óleo contornou os subdomínios $\Omega^{(2)}$ e $\Omega^{(3)}$ e alcançou o poço de ejeção. Em ambos os exemplos o método apresentado captou com precisão e eficiência os fenômenos físicos presentes em problemas de escoamento em meios porosos heterogêneos. Os 
resultados ilustram também a importancia da simulação numérica para modelar problemas com geometrias complexas e forças capilares descontínuas.

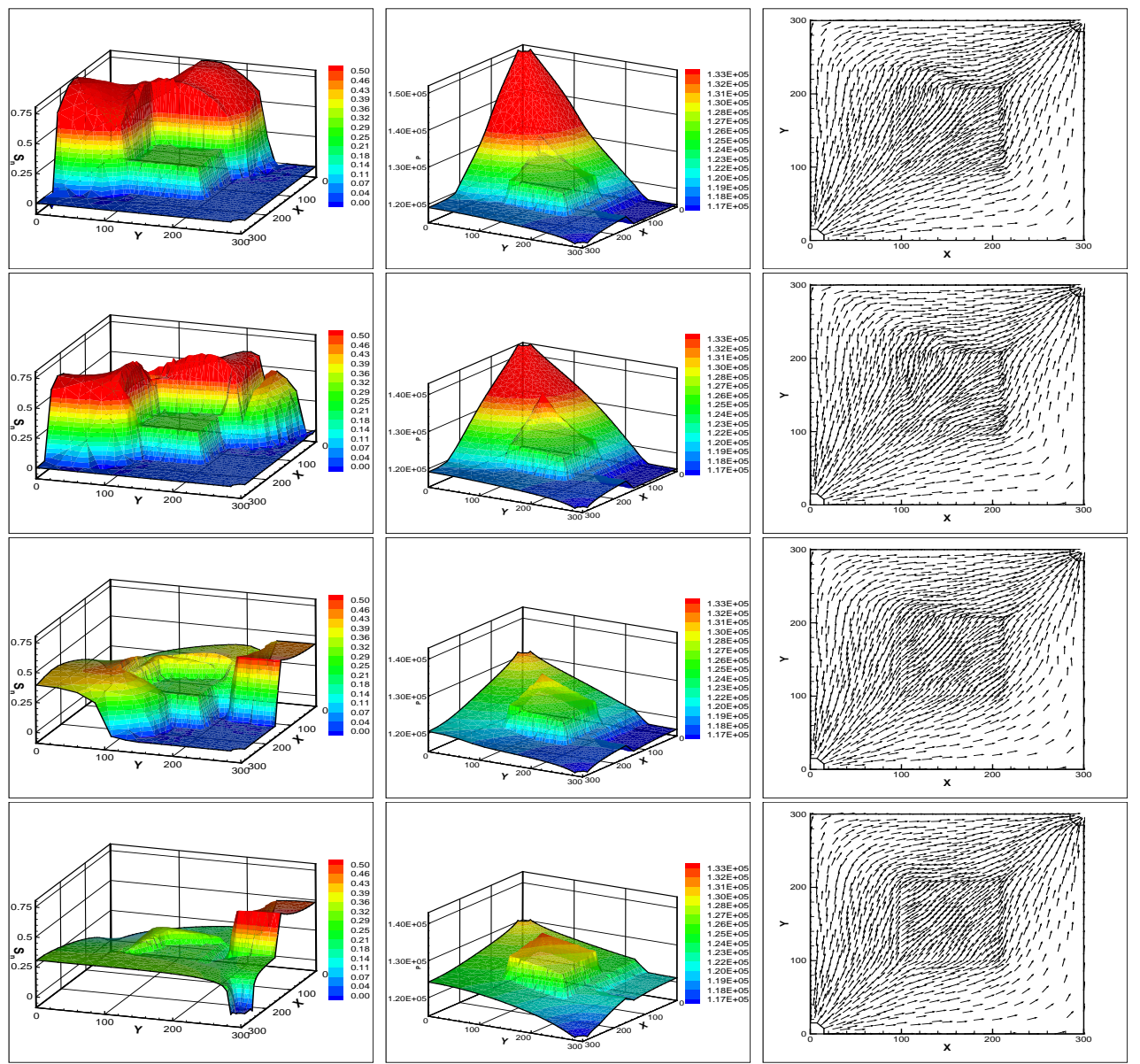

Figura 3: Saturação, pressão global e velocidade total para o Teste 1b. Primeira linha : $t=43$ dias; segunda linha: $t=120$ dias; terceira linha: $t=600$ dias e quarta linha: $t=4150$ dias.

\section{Referências}

[1] M. Bertsch, R. Dal Passo, and C. Van Duijn. Analysis of oil trapping in porous media flow. SIAM J. Math. Anal., 35:245-267, 2003.

[2] D. Di Pietro, A. Ern, and J. Guermond. Discontinous Galerkin methods for anisotropic semidefinite diffusion with advection. SIAM J. Numer. Anal., 46(2):805-831, 2008.

[3] C. J. v. Duijn and M. Neef. Similarity solution for capillary redistribution of two phases in a porous medium with a single discontinuity. Advances in Water Resources, 21:451-461, 1998.

[4] A. Ern, I. Mozolevski, and L. Schuh. Discontinous Galerkin approximation of two-phase flows in heterogeneous porous media with discontinuous capillary pressures. Computer Methods in Applied Mechanics and Engineering, 199:1491-1501, 2010.

[5] A. Ern, A. F. Stephansen, and P. Zunino. A discontinuous Galerkin method with weighted averages for advection-diffusion equations with locally vanishing and anisotropic diffusivity. IMA J. Numer. Anal., 29(2):235-256, 2009. doi: 10.1093/imanum/drm050.

[6] B. G. Ersland, M. S. Espedal, and R. Nybo. Numerical methods for flow in a porous medium with internal boundaries. Computational Geosciences, 2:217-240, 1998.

[7] I. Mozolevski and L. Schuh. Numerical simulation of two-phase immiscible incompressible flows in heterogeneous porous media with capillary barriers. Journal of Computational and Applied Mathematics, 242:12-27, 2013.

[8] C. J. van Duijn, J. Molenaar, and M. Neef. The effect of capillary forces on immiscible two-phase flow in heterogeneous porous media. Transport in Porous Media, 21:71-93, 1995. 